\title{
Weapon carrying: an important part of risk assessment
}

\author{
David W. Perry, Irene D. Cormack, Colin Campbell and Alison Reed
}

\begin{abstract}
Over the past 20 years, with the closure of psychiatric hospltals, there has been a greater emphasis on treatment of the seriousty mentally ill in the community. Recently, there have been untoward incidents involving psychiatric patients loading to increasing public concem over this policy. Steps to recuce this concem have included the care programme approach, the supervision register and the community supervision order. Each requires some form of risk assessment to be carried out prior to their implementation, although there is littile guidance on what areas should be covered.
\end{abstract}

A project set up to develop research-based multidisciplinary care protocols for people suffering with schizophrenia in the community included a comprehensive risk assessment (Cormac, I. D., Perry, D. W. \& Campbell, C. A. in 'Proceedings of the Royal College of Psychiatrists Winter Meeting 1996. p. 44, available upon request from the author). One finding to emerge was that a much greater number of people suffering with schizophrenia than expected carried weapons while unwell and this was felt to warrant further analysis.

\section{The study}

Twenty-five people living in various community settings and fulfilling ICD-10 (World Health Organization, 1992) diagnostic criteria for schizophrenia were included. Each patient was under the care of one consultant and required the care of the multi-professional team. The patients came from a mixed urban and rural catchment area. The cases were selected to field test the protocol for schizophrenia, and consequently were chosen to represent a wide range of patients in terms of age, severity and length of illness. This approximated to $20 \%$ of the patients suffering with schizophrenia under the care of the consultant. None had a history of learning disability or violent offending behaviour.

The age range was 19-65 with a gender distribution of 18 males to five females. Of the 25 patients in the cohort, two declined to give consent to the full risk assessment and therefore analysis was carried out on the remaining 23 .
Each participant underwent an assessment of psychopathology, attitudes to illness, social circumstances, compliance and potential risk. This consisted of existing validated rating scales supplemented by semi-structured interviews and attitude scales, which took four to eight hours to complete over a number of interviews. This was supplemented by assessments with relatives taking approximately a further two hours.

\section{Findings}

Six of the 23 people assessed (26\%) admitted to carrying a weapon while unwell at some time during their illness. This included five men and one woman, their median age being 46. The weapons carried included four knives, one cosh and one screwdriver. In all cases this was an unusual behaviour for the individual, none having carried weapons prior to the onset of their illness. The weapon carrying seemed to be precipitated by psychotic phenomena. Four episodes were secondary to persecutory delusions, one to persecutory hallucinations and one to both. All patients ceased to carry weapons following treatment of the acute episode. However, two of the six resumed weapon carrying during subsequent relapses.

Of those in contact with family members or carers, only two knew of the practice. Three of the six, all men, had a history of substance or alcohol misuse, compared to two in the nonweapon carrying group. All six were compliant with follow-up, although two were only partially compliant with medication because of sideeffects.

\section{Comment}

The prevalence of weapon carrying by the general population, particularly young males, is difficult to determine but is felt to be on the increase. Home office statistics relating to cautions for carrying of offensive weapons show an increase in Warwickshire, and England, from 1990-1994 (Home Office Research and Statistics Directorate. Crime and Criminal Justice Unit, personal 
communication). Why someone carries a weapon when psychotic as opposed to withdrawing socially cannot be interpreted by this study.

However, previous authors have pointed to the nature of psychotic phenomena as being important: Wessely et al (1993) suggested that persecutory delusions were the most likely to be acted upon. Taylor (1985) also noted the association between delusions and violent offending. Interestingly, the majority of those who carried weapons did so in response to persecutory delusions. This has been described as the concept of rationality within irrationality, in that if one suspends concern about the irrationality of psychotic symptoms and accepts that they are experienced as real then attempting to protect oneself is a 'rational' act (Link \& Stueve, 1994).

Although the risk associated with this behavlour in response to psychotic phenomena is unknown, this is clearly a significant finding in a risk assessment particularly with regard to safeguards which need to be taken in the case of relapse.

We feel that the carrying of weapons should be incorporated into any standardised clinical risk assessment. Further work needs to be done on the prevalence of this practice in larger populations.

\section{References}

LINK, B. G. \& STUEve, A. (1994) Psychotic symptoms and the violent/llegal behaviour of mental patients compared to community controls. In Violence and Mental Disorder. Developments in Risk Assessment (eds J. Monahan \& H. J. Steadman), pp. 137-159. Chicago, IL: University of Chicago Press.

TAYLR, P. (1985) Motives for offending among violent and psychotic men. British Joumal of Psychiatry. 147, 491498.

Wessely, S., Buchanan, A., ReAd, A., et al (1993) Acting on Delusions I: Prevalence. Brttish Journal of Psychiatry. 163, 69-76.

WORID HEALTH ORGanization (1992) The Tenth Revision of the International Classification of Diseases and Related Health Problems (ICD-10). Geneva: WHO.

D. W. Perry, Research Registrar in Psychiatry, Black Country Mental Health NHS Trust; Irene D. Cormack, Registrar in Psychiatry. "Colin Campbell, Consultant Psychiatrist, St Michael's Hospital (formerly Central Hospital). St Michael's Road, Warwick CV34 5QW; and Alison Reed, Senior Lecturer in Forensic Psychiatry, Reaside Clinic, Birmingham

*Correspondence 